ies in post-brachytherapy glands may be impaired due to the presence of several false-positive results. In our institution we have no experience with MRI for the detection of local tumor recurrence after HIFU ablation or cryotherapy.

Dr. Adilson Prando

Head, Department of Radiology and Diagnostic Imaging, Vera Cruz Hospital Campinas, São Paulo, Brazil

E-mail: adilson.prando@gmail.com

\title{
PATHOLOGY
}

\section{Prostate-specific antigen kinetics during follow-up are an unreliable trigger for intervention in a prostate cancer surveillance program}

Ross AE, Loeb S, Landis P, Partin AW, Epstein JI, Kettermann A, Feng Z, Carter HB, Walsh PC

Departments of Urology and Pathology, The Johns Hopkins University School of Medicine, The James Buchanan Brady Urological Institute, The Johns Hopkins Hospital, Baltimore, MD, USA

J Clin Oncol. 2010; 28: 2810-6

Purpose: To assess the predictive ability of prostate-specific antigen (PSA) velocity (PSAV) and doubling time (PSADT) for biopsy progression and adverse pathology at prostatectomy among men with low-risk prostate cancer enrolled on an active-surveillance program.

Methods: We evaluated 290 men who met criteria for active surveillance (ie, PSA density $<0.15 \mathrm{ng} / \mathrm{mL} / \mathrm{cm}(3)$ and Gleason score $<$ or $=6$ with no pattern $>$ or $=4$, involving $<$ or $=2$ cores with cancer, and $<$ or $=50 \%$ involvement of any core by cancer) with two or more serial PSA measurements after diagnosis from 1994 to 2008. Follow-up included twice-yearly digital rectal exam and PSA measurements and yearly surveillance biopsy. Treatment was recommended for biopsy progression (ie, Gleason score $>$ or $=7$, or $>2$ positive cores, or $>50 \%$ core involvement). Sensitivity and specificity of postdiagnostic PSAV and PSADT were explored by using receiver operating characteristic (ROC) analysis.

Results: Overall, 188 (65\%) men remained on active surveillance, and 102 (35\%) developed biopsy progression at a median follow-up of 2.9 years. PSADT was not significantly associated with subsequent adverse biopsy findings $(\mathrm{P}=.83)$, and PSAV was marginally significant $(\mathrm{P}=.06)$. No PSAV or PSADT cut point had both high sensitivity and specificity (area under the curve, 0.61 and 0.59 , respectively) for biopsy progression. In those who eventually underwent radical prostatectomy, PSAV $(\mathrm{P}=.79)$ and PSADT $(\mathrm{P}=.87)$ were not associated with the presence of unfavorable surgical pathology.

Conclusion: Postdiagnostic PSA kinetics do not reliably predict adverse pathology and should not be used to replace annual surveillance biopsy for monitoring men on active surveillance.

\section{Editorial Comment}

This is an important study concluding that postdiagnostic PSA kinetics do not reliably predict adverse pathology and should not be used to replace annual surveillance biopsy for monitoring men on active surveillance. At Johns Hopkins, the criteria for active surveillance are: PSA density $<0.15 \mathrm{ng} / \mathrm{mL} / \mathrm{cm} 3$, Gleason score $\leq 6$ with no pattern 4 or 5 , involving $\leq 2$ cores with cancer, and $\leq 50 \%$ involvement of any core by cancer (1). 
At Stanford, the criteria are: Gleason score $\leq 6$ with no pattern 4 or 5 , one single core with cancer, linear extent of cancer $\leq 3 \mathrm{~mm}$, and serum PSA is not considered (2). It is controversial whether percentage or linear extent is the best measure.

The follow-up included twice-yearly digital rectal exam and PSA measurements and yearly surveillance biopsy. Tretament was recommended for biopsy progression which was considered whenever Gleason score was $\geq 7,>2$ positive cores, or $>50 \%$ core involvement. The important finding in Ross et al. study was that PSA double time was not significantly associated with subsequent adverse biopsy findings $(\mathrm{p}=0.83)$ and PSA velocity was marginally significant $(\mathrm{p}=0.06)$.

\title{
References
}

1. Bastian PJ, Mangold LA, Epstein JI, Partin AW: Characteristics of insignificant clinical T1c prostate tumors. A contemporary analysis. Cancer. 2004; 101: 2001-5.

2. Noguchi M, Stamey TA, McNeal JE, Yemoto CM: Relationship between systematic biopsies and histological features of 222 radical prostatectomy specimens: lack of prediction of tumor significance for men with nonpalpable prostate cancer. J Urol. 2001; 166: 104-9; discussion 109-10.

\author{
Dr. Athanase Billis \\ Full-Professor of Pathology \\ State University of Campinas, Unicamp \\ Campinas, São Paulo, Brazil \\ E-mail: athanase@fcm.unicamp.br
}

doi: $10.1590 / S 1677-553820100003000021$

\section{The value of mandatory second opinion pathology review of prostate needle biopsy interpreta- tion before radical prostatectomy}

Brimo F, Schultz L, Epstein JI

Department of Pathology, The Johns Hopkins Hospital Medical Institutions, Baltimore, Maryland, USA

J Urol. 2010; 184: 126-30

Purpose: We determined the value of mandatory second opinion pathology review to interpret prostate needle biopsy before radical prostatectomy.

Materials and Methods: In all cases referred to our institution for radical prostatectomy in 1 year we compared pathological parameters in original and reviewed pathology reports, including benign, atypical or malignant diagnosis, final Gleason score, positive core number, core highest cancer percent and perineural invasion or extraprostatic extension. A major Gleason score discrepancy was defined as a change to a different risk category $(6,7$ and $8-10)$. We defined a significant difference in the highest percent of cancer in a core as $30 \%$ or greater.

Results: Of the 855 cases originally diagnosed as prostatic adenocarcinoma cancer was confirmed in 844 $(98.8 \%)$ by needle biopsy and prostatectomy, of which $9(1 \%)$ were atypical and $2(0.2 \%)$ were benign upon review. A major discrepancy in Gleason score was present in 124 cases (14.7\%), of which 57 (46.0\%) were upgraded and $67(54 \%)$ were downgraded. Of cases with a final Gleason score of $6,8.4 \%$ were originally diagnosed as $7(7.8 \%)$ or $8-10(0.6 \%), 21 \%$ with a final score of 7 had an original score of $6(13.2 \%)$ or $8-10$ $(7.8 \%)$ and 21 of $61(34 \%)$ with a score of $8-10$ were originally diagnosed as 7 or less. There were 80 cases $(64.5 \%)$ of disagreement between scores 6 and 7 . Of the 777 cases with the positive core number in each 